\title{
PET Reconstruction Using a Cooperative Coevolution Strategy in LOR Space
}

\author{
Franck P. Vidal, Jean Louchet, Jean-Marie Rocchisani, and Évelyne Lutton
}

\begin{abstract}
This paper presents preliminary results of a novel method that takes advantage of artificial evolution for positron emission tomography (PET) reconstruction. Fully 3D tomographic reconstruction in $\mathrm{PET}$ requires high computing power and leads to many challenges. To date, the use of such methods is still restricted due to the heavy computing power needed. Evolutionary algorithms have proven to be efficient optimisation techniques in various domains. However the use of evolutionary computation in tomographic reconstruction has been largely overlooked. We propose a computer-based algorithm for fully 3D reconstruction in PET based on artificial evolution and evaluate its relevance.
\end{abstract}

Index Terms-Positron emission tomography, genetic algorithms, optimization methods.

\section{INTRODUCTION AND MOTIVATIONS}

$\mathbf{E}$ VOLUTIONARY algorithms (EAs) have proven to be efficient optimisation techniques in various domains [1], including medicine [2] and medical imagery [3], [4], [5]. However their use in tomographic reconstruction has been largely overlooked. In previous work, we demonstrated that a cooperative coevolution strategy (also called Parisian evolution) called "fly algorithm" [6] could be used in single-photon emission computed tomography (SPECT) reconstruction [7].

For PET, a positron emitter is used as radionuclide for labelling. During the annihilation, it produces two coincidence photons emitted in opposite directions (see Figure 1). Using this property, this radiation is detected in coincidence using a dedicated system. Prior to the reconstruction, the data is often rebinned into a sinogram [8]. This intermediate data representation corresponds to projection data that can be used by conventional tomographic reconstruction codes. The main methods are analytical or based on statistical analysis. These include the most widely used techniques are that based on the maximum-likelihood expectation-maximization method (ML-EM) [9] such as the ordered subset expectationmaximization [10] algorithms. A broad overview of reconstruction methods using sinograms in nuclear medicine can be found in [11]. The trend today is to use more realistic physics-based models, e.g. to correct Compton scattering,

Manuscript received November 13, 2009. This work has been partially funded by Agence Nationale de la Recherche (ANR).

F. P. Vidal is with INRIA Saclay - Ille-de-France/APIS, Orsay, France and with CEA, LIST, Saclay, Gif-sur-Yvette, France and he is member of Fondation Digiteo (http://www.digiteo.fr) (e-mail: franck.p.vidal@gmail.com).

J. Louchet was INRIA Saclay - Île-de-France/APIS, Orsay, France. He is now with Artenia, Châtillon, France (e-mail: jean.louchet@gmail.com).

J.-M. Rocchisani is with Paris XIII University, UFR SMBH \& Avicenne hospital, Bobigny, France (e-mail: jean-marie.rocchisani@avc.aphp.fr).

É. Lutton is with INRIA Saclay - Île-de-France/APIS, Orsay, France (email: evelyne.lutton@inria.fr).

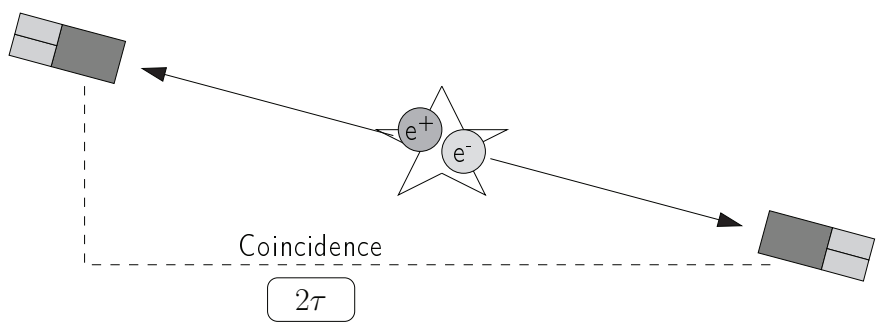

Fig. 1. Photon coincidence detection.

tissue attenuation, and patient's motion. However, the use of such methods is still restricted in the clinical routine due to the heavy computing power needed.

In this paper, we present a novel approach that takes advantage of artificial evolution in list-mode PET reconstruction. It makes use of an ad hoc version of the "fly algorithm". Our aim is to reduce the computation cost, whilst retaining the required quality. Preliminary results, that we present here, seem to be promising and they will lead to further revisions.

The section below presents the main principles of the fly algorithm. Our evolutionary scheme for PET reconstruction is describe in Section III. Section IV shows the results obtained using a numerical phantom. The paper ends with a short conclusion.

\section{PREVIOUS WORK}

Our previous attempt to use a cooperative coevolution scheme based on the fly algorithm was in SPECT [6], [7]. The input data corresponds to raw 2D projections at successive angles around the patient. The projections are then processed to build a sinogram. Each fly represents a point of the patient $3 \mathrm{D}$ space. The final population of flies corresponds to the tracer concentration in the patient who is scanned. Each fly acts as a photon emitter, i.e. it is orthogonally projected onto virtual detectors. Simulated projections are then compared with the real input data, e.g. using a distance metrics. We introduced a "marginal fitness" metrics based on the "leaveone-out cross-validation" method to evaluate the personal contribution of each fly (see Eq. 1) and assess their validity. An artificial evolution loop is then used to optimise the flies' position.

To speed up the reconstruction time and reduce the amount of memory needed by the algorithm, only $3 \%$ of the input data is used at a time by a fly during the reconstruction. Indeed, for each fly, only four orthogonal projections are simulated.

This SPECT reconstruction approach, based on the fly algorithm, gave promising results. PET is considered as a major 


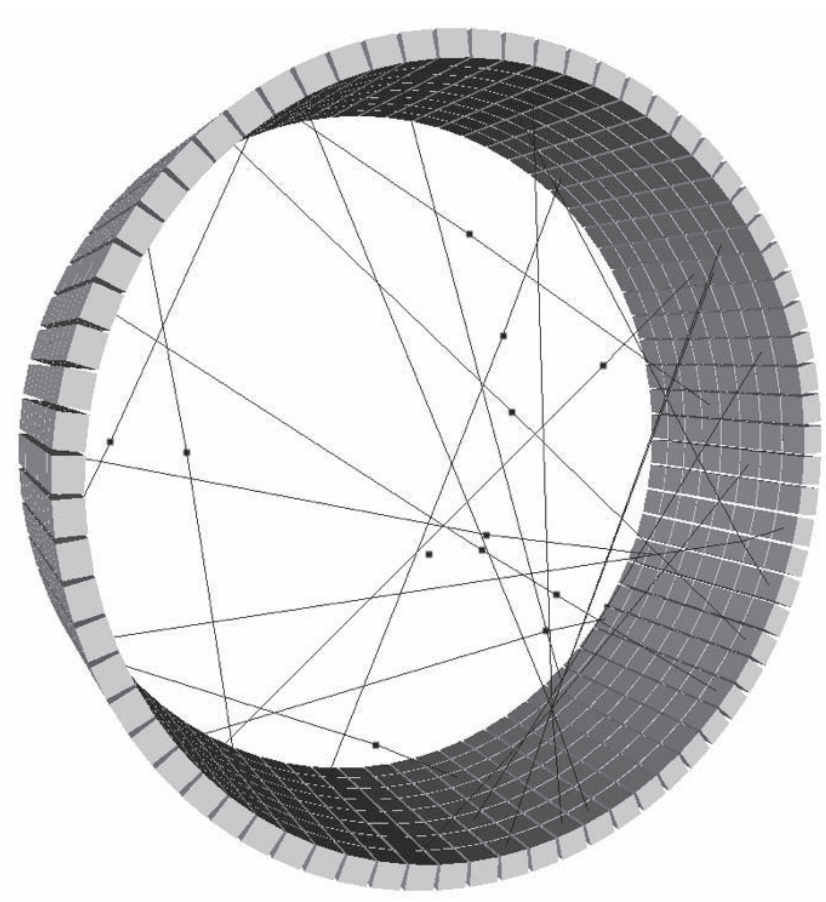

Fig. 2. Each fly represents an emitting point that generates $n$ annihilations.

breakthrough in molecular imaging due to its higher spatial resolution and sensitivity leading to a superior diagnostic accuracy. The section below shows how to make use of the fly algorithm in PET, taking into account the specificity of this imaging modality.

\section{EVOlutionary PET RECONSTRUCTION}

Here again, each fly corresponds to a 3D point, and acts as a radioactive emitter. Their initial positions are randomly generated in the 3D space of the patient. The population of flies will evolve, thanks to genetic operators, to optimise the position of radioactive emitters with respect to the input data. The final population of flies corresponds to the tracer density in the patient, i.e. the reconstructed data. Note that cross-over operations are not used in this application. In our context, the result of such an operation may lead to meaningless results, e.g. in the case of cross-over between two flies of two distinct objects (the new fly will be wrongly located in between). Only mutation and immigration are used.

Each time a new fly is created, a stochastic simulation is performed to compute the fly's illumination pattern. In PET, a set of lines of response (LORs) that correspond to annihilation events can be extracted from the list-mode input data. In the simulation, the scanner properties (e.g. detector blocks and crystals positions) are modelled, and each fly is producing an adjustable number of annihilation events (see Figure 2). Each fly keep a record of its simulated LORs. The result of this simulation consists of a list of pairs of detector identification numbers that correspond to LORs.

The fitness metrics corresponds to a distance measurement between the simulated data and the actual data given by the imaging system. City block distance provides a good

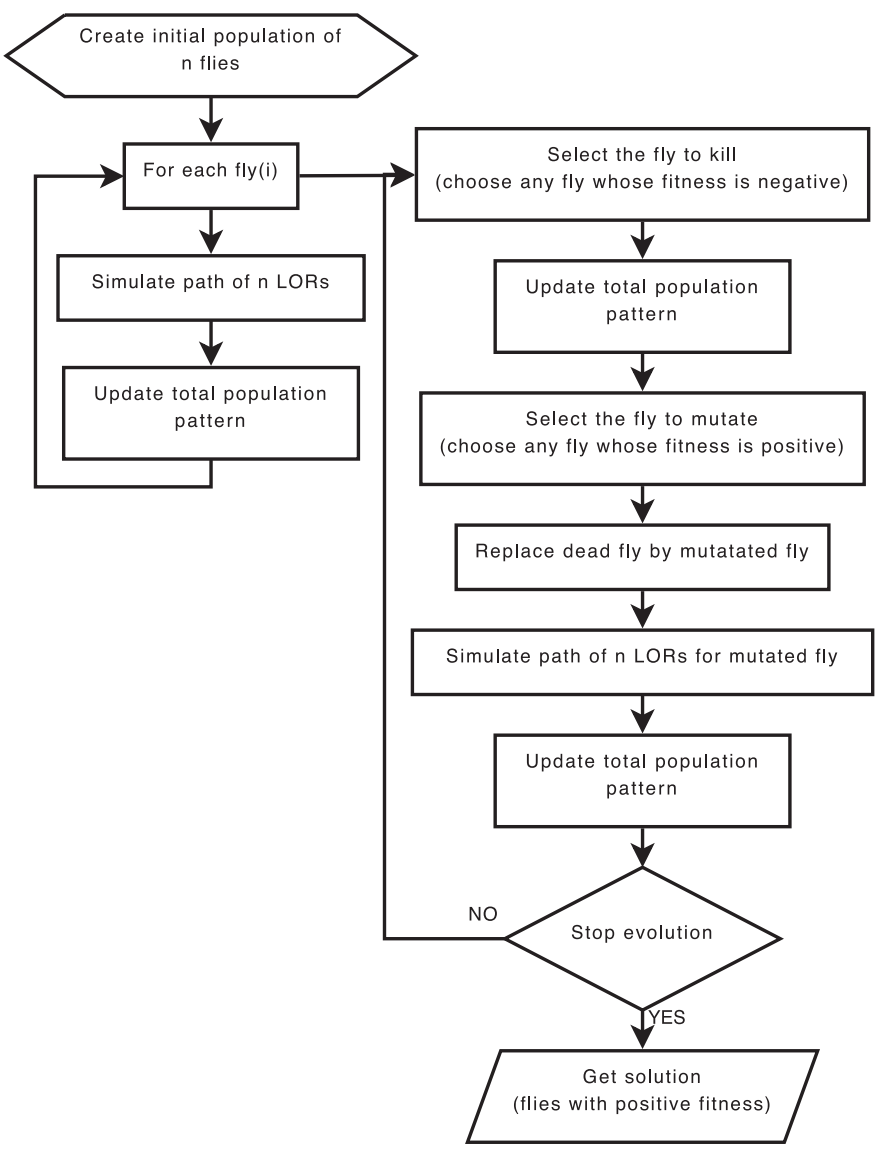

Fig. 3. Evolutionary tomographic reconstruction flow chart.

compromise between accuracy and speed. To assess a given fly $(i)$, a marginal fitness $\left(F_{m}(i)\right)$ based on the leave-one-out cross-validation method is used once again:

$$
\mathrm{F}_{\mathrm{m}}(i)=\operatorname{dist}(\text { pop, input })-\operatorname{dist}(\text { pop }-\{i\}, \text { input })
$$

with $\mathrm{F}_{\mathrm{m}}(i)$ the marginal fitness of Fly $i$, dist $(A, B)$ the city block distance between two tables $A$ and $B$, pop is the set of LORs simulated by the whole population, input is the set of LORs extracted from the input data, and pop $-\{i\}$ is the set of LORs simulated by the whole population without Fly $i$. It gives the personal contribution (positive or negative) of a fly with respect to the whole population. This fitness calculation method is then integrated into an evolution strategy scheme to optimise the position of flies (see Figure 3). In particular, it is used at each iteration of the evolution loop to select the fly to kill, and eventually the fly to mutate. Note that an iteration corresponds to the killing of a 'bad' fly and the creation of a new fly, either by mutation of a 'good' fly or by random immigration. When a fly is killed, its LORs are removed from the total set of simulated LORs. Similarly, when a new fly is created, its LORs are added. This process needs to be fast to be able to decrease the number of bad flies and increase the number of good flies as much as possible.

\section{RESULTS}

We have developed numerical phantom models to assess the reconstruction algorithm. To date, only true coincidence events 
have been considered. Whilst this is not physically correct, it allows us to test and validate our approach in the simplest cases.

The test case that is presented here has been designed to assess the ability of our algorithm to detect small objects, and their relative radioactivity concentrations. Nine cylinders having two different radii $(1 \mathrm{~cm}$ and $2.5 \mathrm{~cm})$ and with five different radioactivity concentrations $\left(C_{1}=114,590 \mathrm{count} / \mathrm{ml}\right.$, $C_{2}=2 C_{1}, C_{3}=3 C_{1}$, etc.) have been simulated (see Figure 4(a)). A low resolution PET system has been simulated. It is made of a single ring of 72 linear blocks that include only 1 crystal.

To evaluate the results, a $512 \times 512$ pixel slice is produced (see Figure 4(b)). Note that the typical image size in PET is $128 \times 128$ pixels. The slices are filtered using a gaussian convolution kernel, then linearly rescaled between zero and one. The reconstructed data appears to be visually close to the input data. In particular, the respective size and concentration of cylinders are well preserved.

To assess the size of cylinders, three horizontal profiles have been extracted so that each cylinder is crossed in its centre (see Figure 5). Full width at half maximum (FWHM) is also measured to quantify errors (see Table I). Let Object $i$ be the cylinder whose concentration is $C_{i}$ in the phantom model and $C_{i}^{\prime}$ in the reconstructed slice. Whilst the profiles in the

TABLE I

FWHM ESTIMATED FROM FIGURE 5

\begin{tabular}{cccc}
\hline Object & $\begin{array}{c}\text { FWHM from } \\
\text { phantom model } \\
\text { (in mm) }\end{array}$ & $\begin{array}{c}\text { FWHM from } \\
\text { slice } \\
\text { (in mm) }\end{array}$ & $\begin{array}{c}\text { Relative } \\
\text { difference } \\
\text { (in \%) }\end{array}$ \\
\hline 1 & 19 & 13 & 32 \\
2 & 49 & 43 & 11 \\
3 & 19 & 14 & 24 \\
4 & 49 & 44 & 10 \\
5 & 19 & 12 & 35 \\
\hline
\end{tabular}

reconstructed slice seem to match respective profiles in the phantom model, error measurements in FWHM are relatively high for the smallest cylinders. The influence of the high resolution of the reconstructed slice with respect to the low spatial resolution of the PET system that has been simulated would need to be investigated.

To assess the validity of the radioactivity concentration within cylinders, the average value at the centre of each cylinder has been measured in Figure 4(b) (see Table II). We

TABLE II

RELATIVE RADIOACTIVITY CONCENTRATION ESTIMATED FROM FIGURE 4(B)

\begin{tabular}{ccc}
\hline Object & $\begin{array}{c}\text { Relative } \\
\text { concentration }\end{array}$ & $\begin{array}{c}\text { Relative error } \\
\text { (in \%) }\end{array}$ \\
\hline 1 & $C_{1}^{\prime}$ & N/A \\
2 & $2.13 \times C_{1}^{\prime}$ & 7.0 \\
3 & $2.67 \times C_{1}^{\prime}$ & 10.1 \\
4 & $3.80 \times C_{1}^{\prime}$ & 5.5 \\
5 & $5.02 \times C_{1}^{\prime}$ & 0.9 \\
\hline
\end{tabular}

compare the respective ratio of the different concentrations to the lower value $\left(C_{1}^{\prime}\right)$, so that we can compare the reconstructed

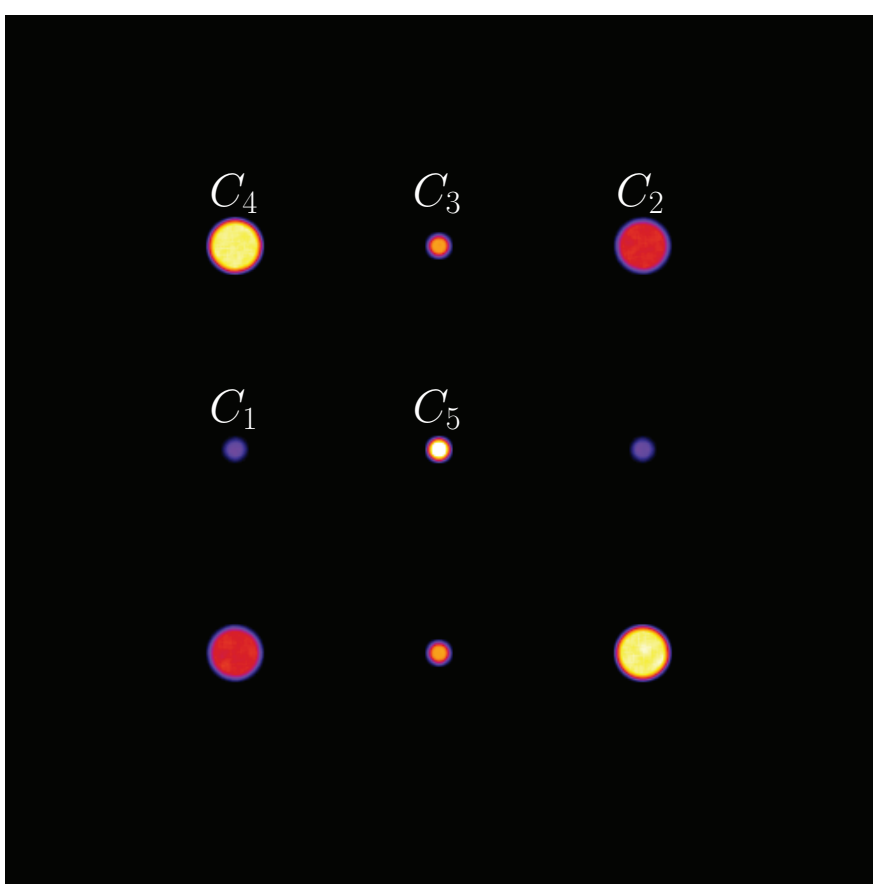

(a) Phantom model.

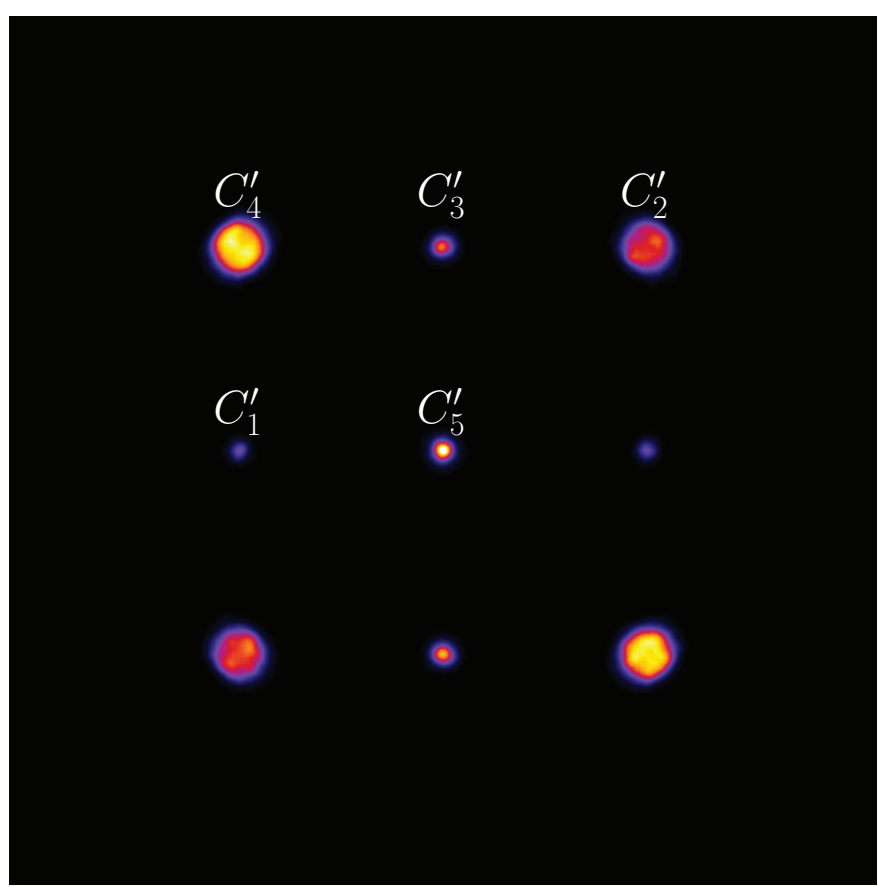

(b) Reconstructed data (flies' fitness $\geq 0$ ).

Fig. 4. Slices $(512 \times 512$ pixels $)$ through the cylinders.

values with the theoretical values. In theory, we should get $C_{2}^{\prime}=2 C_{1}^{\prime}, C_{3}^{\prime}=3 C_{1}^{\prime}$, etc. Table II shows that the relative concentrations have been preserved in the reconstructed slice. However, the maximum relative error is about $10 \%$.

\section{CONCLUSION}

We have presented a novel method for reconstructing tomographic data in PET using evolutionary computation. Our evolutionary scheme is able to reconstruct our test objects. 


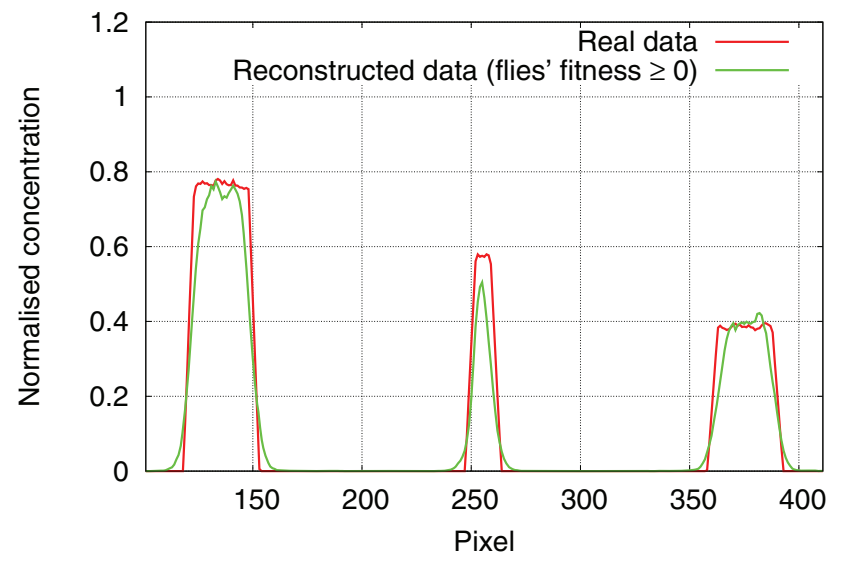

(a) $1^{\text {st }}$ line.

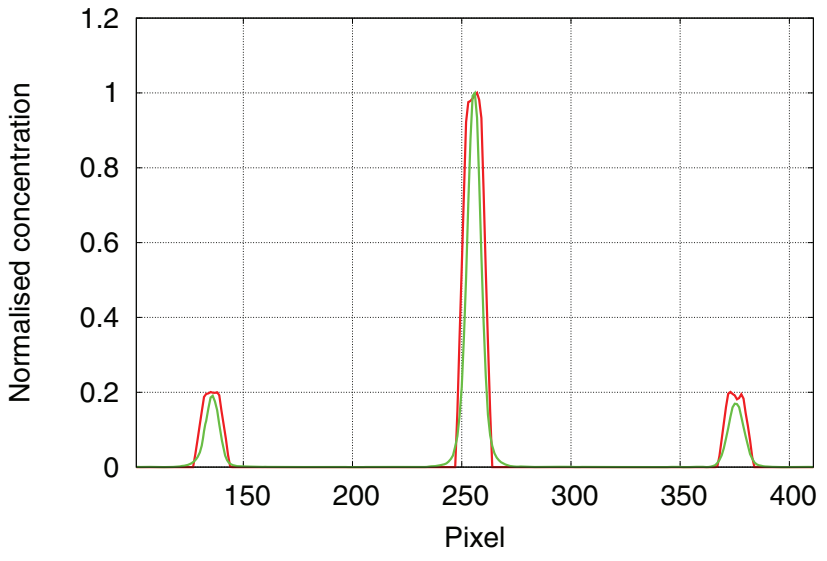

(b) $2^{\text {nd }}$ line.

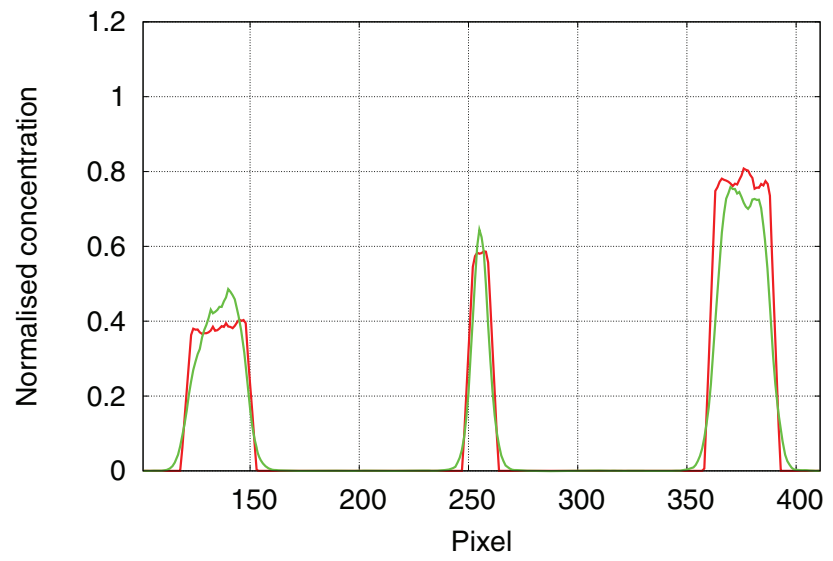

(c) $3^{\text {rd }}$ line

Fig. 5. Profiles extracted from Figure 4.

It provides promising early results that will lead to further refinements. These will include the use of high resolution scanner models, the correction of scattering and attenuation, the use of more realistic input data (including time of fly (TOF)), and a comparison study against ML-EM and/or OSEM.

\section{ACKNOWLEDGMENT}

The authors would like to thanks Dr. Legoupil and Dr Lazaro-Ponthus for their consistent help and relevant comments during the project.

\section{REFERENCES}

[1] G. Olague, S. Cagnoni, and E. Lutton, "Introduction to the special issue on evolutionary computer vision and image understanding," Pattern Recognition Letters, vol. 27, no. 11, pp. 1161-1163, 2006.

[2] C. Pea-Reyes and M. Sipper, "Evolutionary computation in medicine: an overview," Artificial Intelligence in Medicine, vol. 19, no. 1, pp. 1-23, 2000.

[3] P. A. N. Bosman and T. Alderliesten, "Evolutionary algorithms for medical simulations: a case study in minimally-invasive vascular interventions," in Proceedings of the 2005 workshops on Genetic and evolutionary computation (GECCO '05), 2005, pp. 125-132.

[4] S. Cagnoni, A. B. Dobrzeniecki, R. Poli, and J. C. Yanch, "Genetic algorithm-based interactive segmentation of $3 \mathrm{~d}$ medical images," Image and Vision Computing, vol. 17, no. 12, pp. 881-895, 1999.
[5] K. Völk, J. F. Miller, and S. L. Smith, "Multiple network CGP for the classification of mammograms," in EvoWorkshops, ser. Lecture Notes in Computer Science, vol. 5484. Springer, 2009, pp. 405-413.

[6] J. Louchet, "Stereo analysis using individual evolution strategy," in Proceedings of the International Conference on Pattern Recognition (ICPR 'O0), 2000, p. 1908.

[7] A. Bousquet, J. Louchet, and J.-M. Rocchisani, "Fully three-dimensional tomographic evolutionary reconstruction in nuclear medicine," in Artificial Evolution (EA '07), ser. Lecture Notes in Computer Science, vol. 4926, 2007, pp. 231-242.

[8] F. H. Fahey, "Data acquisition in PET imaging," Journal of Nuclear Medicine Technology, vol. 30, no. 2, pp. 39-49, 2002.

[9] L. A. Shepp and Y. Vardi, "Maximum likelihood reconstruction for emission tomography," IEEE Transactions on Medical Imaging, vol. 1, no. 2, pp. 113-122, 1982.

[10] H. M. Hudson and R. S. Larkin, "Accelerated image reconstruction using ordered subsets of projection data," IEEE Transactions on Medical Imaging, vol. 13, no. 4, pp. 601-609, 1994.

[11] R. M. Lewitt and S. Matej, "Overview of methods for image reconstruction from projections in emission computed tomography," Proceedings of IEEE, vol. 91, no. 10, pp. 1588-1611, 2003. 